\title{
Circular Polarization of Gamma Rays and Parity Nonconserving Interactions*
}

\author{
O. Dumitrescu * * \\ Institut für Theoretische Physik, Ruhr-Universität Bochum, W-Germany \\ M. GARI *** \\ California Institute of Technology Pasadena, California, USA \\ H. KüMmel and J. G. Zabolitzky \\ Institut für Theoretische Physik, Ruhr-Universität Bochum, W-Germany \\ (Z. Naturforsch. 27 a, 733-740 [1972] ; received 16 December 1971)
}

\begin{abstract}
An explicit expression for the circular polarization of gamma rays emitted by odd mass deformed nuclei is given. A semi-microscopic description of the nuclear excited states based on pairing plus quadrupole and octupole model is used. The average field is based on a deformed diffuse edge potential. The short-range correlations are taken into account via the generalized Bethe-Goldstone-equation.
\end{abstract}

\section{Introduction}

Recently we have calculated one of the most thoroughly investigated parity violating effects in nuclei, the circular polarization of the $\gamma$ line in ${ }^{181} \mathrm{Ta},{ }^{175} \mathrm{Lu}$ and ${ }^{177} \mathrm{Lu}{ }^{1,2}$. In this calculations we have used for the initial and final states the best wave functions that are available today. The parity nonconserving potentials (PNC) used were based on one $\pi$ - and one $\varrho$-exchange (Fig. $1 \mathrm{a}, \mathrm{b}$ ) for all

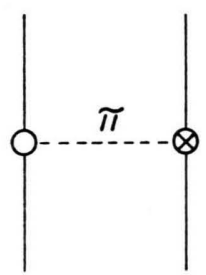

a

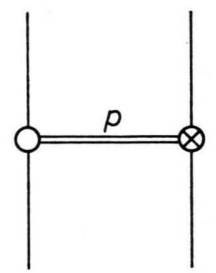

$b$
Fig. 1. Processes taken into account in the calculations of parity mixing ${ }^{1,2}$. The open circle stands for the strong, the cross for the weak interaction.

weak interaction theories en vogue. Effects of short range correlations (SRC) have been taken into account as exactly as possible ${ }^{3,4}$. The results (due to admixtures only) obtained for various strong and weak N-N-potentials are far away from agreement with most other calculations done up to now (see Refs. quoted in 1,4 ). No weak interaction theory

* This work was supported in part by Deutsche Forschungsgemeinschaft and by US AEC AT [04-3]-63

** Permanent address: Chemistry Faculty, Bucharest University, Bucharest, Romania. At present: Alexander von Humboldt fellow. seems to be able to explain the experimental values. At this point one should remind that diagram Fig.1a gives no contribution in exact $\mathrm{SU}(2)$ (in taking into account isospin-breaking only with a negligible amplitude ${ }^{5}$ concerning the strangeness conserving currents). So the only contribution entering with the $\cos ^{2} \theta$ factor is due to $\varrho$-exchange and therefore is highly affected by short range correlations.

Recently we found, however, that calculations of irregular transitions through parity mixing (PNC) are not gauge-invariant ${ }^{6}$. Gauge invariance requires that processes of direct photo emission (PNE), such as Fig. (2a and b) (and additional) also must be included. These processes were shown (in contrast to process Fig. 1a) not to vanish, even in exact $\mathrm{SU}(2)$, which means that the one $\pi$-exchange contribution can enter with the $\cos ^{2} \theta$ factor in the

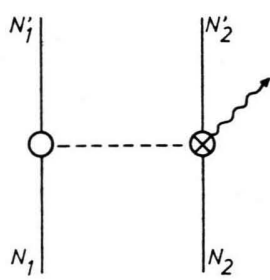

a

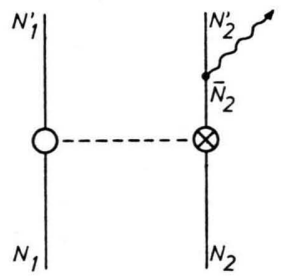

$b$
Fig. 2. Some PNE contributions $\mathrm{N}_{1} \mathrm{~N}_{2} \rightarrow \mathrm{N}_{1}{ }^{\prime} \mathrm{N}_{2}^{\prime} \gamma$ of one $\pi$ (and $\varrho$ )-exchange processes. There appear the same terms with strong and weak vertices exchanged plus some more ${ }^{6}$.

*** Permanent address: Institut für Theoretische Physik, Ruhr-Universität Bochum, W-Germany.

Reprint requests to Dr. H. KüMMEL, Institut für Theoretische Physik II, Ruhr-Universität Bochum, D-4630 Bochum, Buscheystraße, Postfach 2148, W-Germany. 
calculations. These findings have shown that calculations of parity-violating effects in Gammatransitions done up to now 7 are incomplete because of non-gauge-invariant description. It is expected that a complete gauge-invariant theory will improve the situation. A full description of the PNE-operators and their relative importance will be given in ${ }^{8}$.

In the present paper we give explicit expressions for the polarization of gamma lines including all important terms and using the most sophisticated wave functions as applied in 1,2 .

\section{Circular Polarization}

The electromagnetic interaction contains two terms

$$
V_{\mathrm{em}}=V_{\text {reg }}+V^{\mathrm{PNE}}
$$

where $V_{\text {reg }}$ gives the usual amplitude of the transition probability in the framework of the parity conserving theories. The term $V^{\mathrm{PNE}}$ violates the parity law and has been obtained in 6,8 .

The circular polarization can be written as follows $P=-\left\{\sum_{\lambda}\left(e_{\lambda}^{*} m_{\lambda}+m_{\lambda}^{*} e_{\lambda}\right)\right\}\left\{\sum_{\lambda}\left(\left|e_{\lambda}\right|^{2}+\left|m_{\lambda}\right|^{2}\right)\right\}^{-1}$

with

$$
\begin{aligned}
& e(m)_{\lambda}\left(I_{1} K_{1} \rightarrow I_{2} K_{2}\right) \\
& \quad=\mathscr{N}_{\lambda}(k) \hat{I}_{1}^{-1}\left\langle I_{2} K_{2}\|\mathscr{M}\{E(M) \lambda\}\| I_{1} K_{1}\right\rangle \\
& \left(\mathcal{N}_{\lambda}(k)\right)^{2}=8 \pi(\lambda+1) k^{2 \lambda+1}(\hbar \lambda[(2 \lambda+1) ! !])^{-1} .
\end{aligned}
$$

Assuming the validity of the Bohr-Mottelson strong coupling model $^{9}$ the total nuclear wave function factorizes into a rotational and an intrinsic part

$$
\begin{aligned}
|I K M\rangle & =\left((2 I+1) / 16 \pi^{2}\left(1+\delta_{K 0}\right)\right)^{1 / 2} . \\
\cdot & \left(\mathscr{D}_{M K}^{I}|K\rangle+(-)^{I-K} \mathscr{D}_{M-K}^{I}|-K\rangle\right) .
\end{aligned}
$$

Here $I, K$ and $M$ stand for the total nuclear spin and its projections onto the nuclear and laboratory $Z$-axes. The internal motion is described by $|K\rangle$ (and time reversed $|-K\rangle$ ) wave functions.

The reduced matrix element from (3) in this case becomes

$$
\begin{aligned}
\left\langle I_{2} K_{2} \|\right. & \left.\mathscr{M}_{\lambda} \| I_{1} K_{1}\right\rangle \\
= & A_{\lambda}\left(\begin{array}{l}
I_{1} K_{1} \\
I_{2} K_{2}
\end{array}\right)\left\{C_{K_{1} K_{2}-K_{1} K_{2}}^{I_{1}}\left\langle K_{2}\left\|\mathscr{M}_{\lambda}\right\| K_{1}\right\rangle \quad(6)\right. \\
& \left.+(-)^{I_{1}-K_{1}} C_{-K_{1} K_{2}+K_{1} K_{2}}^{I_{2}}\left\langle K_{2}\left\|\mathscr{M}_{\lambda}\right\|-K_{1}\right\rangle\right\}
\end{aligned}
$$

with

$$
A_{\lambda}\left(\begin{array}{l}
I_{1} K_{1} K_{2} \\
I_{2}
\end{array}\right)=\left(1+\delta_{K_{1} 0}\right)^{-1 / 2} \hat{I}_{2}^{-1} \hat{I}_{1} .
$$

The last term in (6) is zero for $\lambda<K_{1}+K_{2}$.
Since the parity nonconserving nucleon-nucleon potential $V^{\mathrm{PNC}}$ is extremely small, the wave functions $|K\rangle$ can be expanded as follows

with

$$
|K\rangle=|K \pi\rangle+\sum_{s}|K-\pi, s\rangle F_{s}
$$

$$
F_{s}=\left(E_{K \pi}-E_{s}\right)^{-1}\left\langle K-\pi, S\left|V_{(12)}^{\mathrm{PNC}}\right| K \pi\right\rangle .
$$

The multipole moments are [see Eq. (1)]

$$
\mathscr{M}_{\lambda \Omega} \equiv \mathscr{M}\{E(M) \lambda\}_{\Omega}=\mathscr{M}_{\lambda, \Omega}^{\mathrm{reg}}+\mathscr{M}_{\lambda,}^{\mathrm{PNE}}
$$

where the first term describes the allowed parity transitions, the second term the irregular ones. Explicit expressions for $M^{\mathrm{PNE}}$ are given in 6,8 .

Hence, for a parity forbidden transition the $\left\langle K_{2}\left\|\mathscr{M}_{\lambda}\right\| K_{1}\right\rangle$ matrix element has the form

$$
\begin{aligned}
\left\langle K_{2} \|\right. & \left.\mathscr{M}_{\lambda \Omega} \| K_{1}\right\rangle=\left\langle K_{2} \pi_{2}\left\|\mathscr{M}_{\lambda,}^{\mathrm{PNE}}\right\| K_{1} \pi_{1}\right\rangle \\
& +\sum_{s} F_{s}^{(1)}\left\langle K_{2} \pi_{2}\left\|\mathscr{M}_{\lambda, \Omega}^{\mathrm{reg}}\right\| K_{1}-\pi_{1}, s\right\rangle \\
& +\sum_{\nu} F_{v}^{(2)}\left\langle K_{2}-\pi_{2}, v\left\|\mathscr{M}_{\lambda, \Omega}^{\mathrm{reg}}\right\| K_{1}, \pi_{1}\right\rangle .
\end{aligned}
$$

For the methods to compute these regular matrix elements see ${ }^{10,11 .}$

The PNC and PNE matrix elements in the case of an odd mass deformed nucleus are of the following form:

$\left\langle K^{\prime} \pi^{\prime} \mid \mathcal{O}(12) \| K \pi\right\rangle=\left\langle\psi_{0}\left|\left[\Omega\left(K^{\prime} \pi^{\prime}\right), \Phi_{K \pi}^{+}\right]-\right| \psi_{0}\right\rangle$

where

$\Phi_{K \pi}^{+}=\frac{1}{2} \sum_{\substack{\tau \tau v^{\prime} \sigma \sigma^{\prime} \\ \nu v^{\prime} \omega \omega^{\prime}}} \mathcal{O}_{\tau \tau^{\prime} \sigma \sigma^{\prime}}^{\left(\nu v^{\prime} \mid \omega \omega^{\prime}\right)} a_{\imath \tau}^{+} a_{\nu^{\prime} \tau^{\prime}}^{+} a_{\omega^{\prime} \sigma^{\prime}} a_{\omega \sigma} \Omega^{+}(K \pi)$

with

$$
\begin{aligned}
& \mathcal{O}_{\tau \tau^{\prime} \sigma \sigma^{\prime}}\left(v v^{\prime} \mid \omega \omega^{\prime}\right) \\
& \quad=\left\langle\chi_{v \tau} \chi_{v^{\prime} \tau^{\prime}}\left|e^{S_{\mathrm{A}^{+}}} \mathcal{O}(12) e^{S_{\mathrm{A}}}\right| \chi_{\omega \sigma} \chi_{\omega^{\prime} \sigma^{\prime}}\right\rangle
\end{aligned}
$$

and $6,8,12$

$$
\mathcal{O}(12)=V^{\mathrm{PNC}}(12) \quad \text { or } \quad \mathscr{M}_{\lambda \Omega}^{\mathrm{PNE}}(12) .
$$

By $\chi_{s \sigma}=a_{s \sigma}^{+}|0\rangle$ we have denoted the s.p. wave functions defined by the independent particle model

$$
H_{\mathrm{av}}=\sum_{s \sigma} \varepsilon_{s} a_{s \sigma}^{+} a_{s \sigma}
$$

based on the deformed diffuse edge potential ${ }^{13}$ (s label all good quantum numbers except the $\operatorname{sign} \sigma= \pm 1$ of the nucleon angular momentum on the nuclear symmetry axis).

The wave functions $|K \pi\rangle$ are solutions of an independent particle model plus effective residual interactions of pairing and long range types 10,11 together with short range correlations. Therefore, 
we construct them as follows

$$
|K \pi\rangle=e^{S_{\mathrm{A}}}\left|\psi_{K} \boldsymbol{\pi}\right\rangle
$$

with $^{10,11}$

$\left(H_{\text {av }}+H_{\text {Pair }}+H_{\text {long range }}-E_{K \pi}\right)\left|\psi_{K \pi}\right\rangle=0$,

where

$$
\begin{aligned}
H_{\text {Pair }} & =-\sum_{\varrho} G_{\varrho} P_{\varrho} ; \quad P_{\varrho}=\sum_{s} a_{s+}^{+} a_{s-}^{+}, \\
H_{\text {long range }} & =-\frac{1}{2} \sum_{\lambda=2,3} \sum_{\mu \geqq 0} \sum_{\varrho, \varrho^{\prime}} X_{\varrho \varrho^{\prime}}^{(\lambda)} q_{\lambda \mu}^{+}(\varrho) q_{\lambda \mu}\left(\varrho^{\prime}\right),
\end{aligned}
$$

defining the multipole momentum matrix elements as follows 11

$$
\begin{aligned}
q_{\lambda \mu}(\varrho) & =\sum_{s s^{\prime}} \sum_{\sigma \sigma^{\prime}}\left\langle s \sigma\left|\frac{r^{\lambda}\left(Y_{\lambda \mu}+(-)^{\mu} Y_{\lambda-\mu}\right)}{\sqrt{2\left(1+\delta_{\mu 0}\right)}}\right| s^{\prime} \sigma^{\prime}\right\rangle a_{s \sigma}^{+} a_{\delta^{\prime} \sigma^{\prime}} \\
& \equiv \sum_{s s^{\prime} \sigma}\left(f\left(s s^{\prime}\right) a_{s \sigma}^{+} a_{s^{\prime} \sigma}+\bar{f}\left(s s^{\prime}\right) a_{s \sigma}^{+} a_{s^{\prime}-\sigma}\right) .
\end{aligned}
$$

By $\varrho$ we have denoted the neutron (n) and proton (p) characteristics, $G_{\varrho}$ and $X_{\varrho \varrho^{\prime}}^{(\lambda)}$ being the corresponding strength parameters.

The solutions $\left|\psi_{K \pi}\right\rangle$ may be written in terms of quasiparticle

$$
\alpha_{s \sigma}^{+}=u_{s} a_{s-\sigma}^{+}+\sigma v_{s} a_{s \sigma}
$$

and phonon operators

$$
\begin{aligned}
Q_{i}^{+}(\lambda \mu)= & \frac{1}{2} \sum_{s s^{\prime}}\left(\psi_{s s^{\prime}}^{i} A^{+}\left(s s^{\prime}\right)-\varphi_{s s^{\prime}}^{i} A\left(s s^{\prime}\right)\right. \\
& \left.+\bar{\psi}_{s s^{\prime}}^{i} \bar{A}^{+}\left(s s^{\prime}\right)-\bar{\varphi}_{s s^{\prime}}^{i} \bar{A}\left(s s^{\prime}\right)\right), \\
Q_{i}(\lambda \mu)\left|\psi_{0}\right\rangle & =0 ; A\left(s s^{\prime}\right)=2^{-1 / 2} \sum_{\sigma} \sigma \alpha_{s \sigma} a_{s^{\prime}-\sigma} ; \\
\bar{A}\left(s s^{\prime}\right) & =2^{-1 / 2} \sum_{\sigma} \alpha_{s \sigma} \alpha_{s^{\prime} \sigma}
\end{aligned}
$$

as follows ${ }^{10}$ :

$$
\left|\psi_{K \pi}\right\rangle=\Omega^{+}(K \pi)\left|\psi_{0}\right\rangle,
$$

with

$$
\begin{aligned}
\Omega^{+}(K \pi) & =2^{-1 / 2} N\left(s_{1} s_{2} \ldots s_{n}\right) \sum_{\sigma}\left\{\sum_{i=1}^{n} C_{s_{i}} \alpha_{s_{i} \sigma}^{+}\right. \\
& \left.+\sum_{\lambda \mu_{i} \nu} \Delta_{\nu \sigma}^{\lambda \mu_{i}}\left(s_{1} s_{2} \ldots s_{n}\right) \alpha_{\nu \sigma}^{+} Q_{i}^{+}(\lambda \mu)\right\} .
\end{aligned}
$$

In treating short range correlations [Eq. (17)] we may neglect three and more particle correlations as not so important in the effect we are interested in 1 , $3,12,14$, i.e. we use the approximation $S_{\mathrm{A}} \rightarrow S_{2 \mathrm{~A}}$ with

$$
S_{2 \mathrm{~A}}=\frac{1}{4} \sum_{\varrho_{1}} \sum_{\varrho_{2}} \sum_{v_{1}} a_{\varrho_{2}}^{+} a_{\varrho_{2}}^{+} a_{v_{1}} a_{v_{2}}\left\langle\varrho_{1} \varrho_{2}\left|S_{2 \mathrm{~A}}\right| v_{2} v_{1}\right\rangle
$$

where here $\nu_{i}$ and $\varrho_{i}$ denote the occupied, respectively unoccupied particle levels of $\left|\psi_{K \pi}\right\rangle$ [see Eq. (24)]. This method is rather good in spite of the fact that in the neighbourhood of the Fermi sea about 50 levels are only partially occupied because of pairing plus long range terms [see Eq. (18)].

\section{PNC-matrix Elements}

Taking into account Eqs. (9), (12) and (25) we obtain

$\left\langle K-\pi\left|V^{\mathrm{PNC}}(12)\right| K \pi\right\rangle$

$=N\left(s_{1} s_{2} \ldots s_{n}\right)\left\{\sum_{i=1}^{n} C_{s_{i}} M_{s_{i} \nu}+\sum_{\lambda \mu_{i} \delta} \Delta_{s}^{\lambda \mu_{i}}\left(s_{1} s_{2} \ldots s_{n}\right) M_{s \nu}^{\lambda \mu_{i}}\right\}$

with

$$
\begin{gathered}
M_{s_{i} \nu} \cong 2^{-1 / 2} \sum_{\sigma}\left\langle\psi_{0}\left|\left[\alpha_{s_{i} \sigma}, \Phi_{K \pi}^{+}(v)\right]\right| \psi_{0}\right\rangle, \\
M_{s \nu}^{\lambda \mu_{i}} \cong 2^{-1 / 2} \sum_{\sigma}\left\langle\psi_{0}\left|\left[Q_{i}(\lambda \mu) \alpha_{s \sigma}, \Phi_{K \pi}^{+}\right]\right| \psi_{0}\right\rangle .
\end{gathered}
$$

Here and in the following we have neglected the contribution of $\bar{f}\left(s s^{\prime}\right)$ [see Eq. (21)] in the $\Delta$-coefficients, i.e. we assumed that they do not depend on the sign $\sigma$.

Applying the method of approximate second quantization ${ }^{15}$ we obtain

for Eq. (28) and

$$
\begin{gathered}
M_{s i v} \cong \sum_{a}\left\{v_{a}^{2}\left(v_{v} v_{s_{i}}-u_{v} u_{s_{i}}\right) \mathscr{A}_{s_{i} a}\left[V_{++,++}^{\mathrm{PNC}}\left(a v \mid s_{i} a\right)+V_{+-}^{\mathrm{PNC}}+-\left(a v \mid s_{i} a\right)\right]\right. \\
\left.+u_{a} v_{a}\left(u_{\nu} v_{s_{i}}+u_{s_{i}} v_{\nu}\right) \mathscr{A}_{a a} V_{+-,+-}^{\mathrm{PNC}}\left(a a \mid v s_{i}\right)\right\}
\end{gathered}
$$

$$
\begin{aligned}
& M_{s \nu}^{\lambda \mu i} \cong 2^{-1 / 2} \sum_{a b}\left\{\left[\left(\psi_{a b}^{i} u_{a} v_{b}+\varphi_{a b}^{i} v_{a} u_{b}\right) u_{s} u_{\nu}+\left(\psi_{a b}^{i} u_{b} v_{a}+\varphi_{a b}^{i} v_{b} u_{a}\right) v_{s} v_{\nu}\right]\right. \\
& \times \mathscr{A}_{a s}\left[V_{++,++}^{\mathrm{PNC}}(s a \mid v b)+V_{-+,-+}^{\mathrm{PNC}}(s a \mid v b)\right]+\left[\left(\psi_{a b}^{i} u_{a} v_{b}+\varphi_{a b}^{i} v_{a} u_{b}\right) u_{s} u_{v}\right. \\
& \left.-\left(\psi_{v b}^{i} u_{b} v_{a}+\varphi_{a b}^{i} v_{b} u_{a}\right) v_{s} v_{v}\right] \mathscr{A}_{a s}\left[V_{-+,++}^{\mathrm{PNC}}(s a \mid v b)+V_{++,-+}^{\mathrm{PNC}}(s a \mid v b)\right] \\
& +\left[\left(\psi_{a b}^{i} u_{a} u_{b}-\varphi_{a b}^{i} v_{a} v_{b}\right) v_{s} u_{v}+\left(\psi_{a b}^{i} v_{a} v_{b}-\varphi_{a b}^{i} u_{a} u_{b}\right) u_{s} u_{v}\right] \\
& \times \mathscr{A}_{s v}\left[V_{+-,++}^{\mathrm{PNC}}(a b \mid v s)-V_{+-,--}^{\mathrm{PNC}}(a b \mid v s)\right]+\left[\left(\psi_{a b}^{i} u_{a} u_{b}-\varphi_{a b}^{i} v_{a} v_{b}\right) v_{s} u_{v}\right. \\
& \left.-\left(\psi_{a b}^{i} v_{a} v_{b}-\varphi_{a b}^{i} u_{a} u_{b}\right) u_{s} u_{v}\right] \mathscr{A}_{s v}\left[V_{+-,-+}^{\mathrm{PNC}}(a b \mid \boldsymbol{v} s)-V_{+-,+-}^{\mathrm{PNC}}(a b \mid \boldsymbol{v} s)\right] \\
& +\psi_{v b}^{i} v_{a}^{2}\left(v_{b} u_{s}+u_{b} v_{s}\right) \mathscr{A}_{a s}\left[V_{++,++}^{\mathrm{PNC}}(a s \mid b a)+V_{+-,-+}^{\mathrm{PNC}}(a s \mid b a)\right] \\
& +\varphi_{s b}^{i} v_{a}^{2}\left(v_{b} u_{v}+u_{b} v_{v}\right) \mathscr{A}_{a v}\left[V_{++,++}^{\mathrm{PN} \dot{\mathrm{C}}}(a b \mid v a)+V_{+-,++}^{\mathrm{PNC}}(a b \mid v a)\right] \\
& +\psi_{v b}^{i} u_{a} v_{a}\left(u_{b} u_{s}-v_{b} v_{s}\right) \mathscr{A}_{s b}\left[V_{-+,+-}^{\mathrm{PNC}}(s b \mid a a)-V_{+-,-+}^{\mathrm{PNC}}(s b \mid a a)\right] \\
& \left.+\varphi_{s b}^{i} u_{a} v_{a}\left(u_{b} u_{v}-v_{b} v_{v}\right) \mathscr{A}_{v b}\left[V_{+-,-+}^{\mathrm{PNC}}(a a \mid v b)-V_{+-,+-}^{\mathrm{PNC}}(a a \mid v b)\right]\right\}
\end{aligned}
$$


where for example

$$
\begin{aligned}
& \mathscr{A}_{v v^{\prime}} V_{\tau \tau^{\prime} \sigma \sigma^{\prime}}^{\mathrm{PNC}}\left(v v^{\prime} \mid \omega \omega^{\prime}\right)=V_{\tau \tau^{\prime} \sigma \sigma^{\prime}}^{\mathrm{PNC}}\left(v v^{\prime} \mid \omega \omega^{\prime}\right)-V_{\tau^{\prime} \tau \sigma \sigma^{\prime}}^{\mathrm{PNC}}\left(v v^{\prime} \mid \omega \omega^{\prime}\right), \\
& \mathscr{A}_{\omega \omega^{\prime}} V_{\tau \tau^{\prime} \sigma \sigma^{\prime}}^{\mathrm{PNC}}\left(v v^{\prime} \mid \omega \omega^{\prime}\right)=V_{\tau \tau^{\prime} \sigma \sigma^{\prime}}^{\mathrm{PNC}}\left(v v^{\prime} \mid \omega \omega^{\prime}\right)-V_{\tau \tau^{\prime} \sigma^{\prime} \sigma}^{\mathrm{PNC}}\left(v v^{\prime} \mid \omega^{\prime} \omega\right) .
\end{aligned}
$$

Here we have assumed that the vibrational states of the double-even nucleus

$$
|\lambda \mu i\rangle \equiv Q_{i}^{+}(\lambda \mu)\left|\psi_{0}\right\rangle
$$

are strongly collective (the individual amplitudes $\psi_{s s^{\prime}}^{i}, \varphi_{s s^{\prime}}^{i}, \bar{\psi}_{s s^{\prime}}^{i}$ and $\bar{\varphi}_{s s^{\prime}}^{i}$ from Eq. (23) are small quantities), i.e. the condition

$$
\left[\alpha_{s \sigma}, Q_{i}(\lambda \mu)\right] \cong 0
$$

is fulfilled. This implies that all terms which take out a quasiparticle from the phonon state must be neglected (in any case there are not many of them) and therefore the matrix element (31) contains only terms which describe the quasiparticle or phonon transitions or a mixture of them.
The $V_{\tau \tau^{\prime} \sigma \sigma^{\prime}}^{\mathrm{PrC}}\left(\boldsymbol{v} v^{\prime} \mid \omega \omega^{\prime}\right)$ should be calculated by transforming to the center of mass and relative motion coordinates, then applying the $e^{S}$-theory ${ }^{1,3}$ $12,14,16$.

\section{PNE-matrix Elements}

Taking into account Eqs. (11), (12) and (25), however neglecting the collective part ${ }^{17}$ in the last eq., i.e.

$$
\begin{aligned}
& \left|K_{1} \pi_{1}\right\rangle \cong 2^{-1 / 2} \sum_{\sigma_{1}} \alpha_{s_{1} \sigma_{1}}^{+}\left|\psi_{0}\right\rangle, \\
& \left|K_{2} \pi_{2}\right\rangle \cong 2^{-1 / 2} \sum_{\sigma_{2}} \alpha_{s_{2} \sigma_{2}}^{+}\left|\psi_{0}\right\rangle,
\end{aligned}
$$

we obtain [see Eqs. (12)-(15)]

$$
\begin{aligned}
& \left\langle K_{2} \pi_{2}\left\|\mathscr{M}_{\lambda, 2}^{\mathrm{PNE}}\right\| K_{1} \pi_{1}\right\rangle \cong 2^{-1} \sum_{\sigma_{1} \sigma_{2}} \sum_{a \sigma}\left\{\sigma_{1} \sigma_{2} v_{s_{1}} v_{s_{2}} v_{a}^{2} \mathscr{A}_{a s_{2}}\left(\mathscr{M}_{\lambda,}^{\mathrm{PNE}}\right)_{\sigma \sigma_{1}, \sigma_{2} \sigma}\left(a s_{1} \mid s_{2} a\right)\right. \\
& \quad-u_{s_{1}} u_{s_{2}} v_{a}^{2} \mathscr{A}_{a s_{2}}\left(\mathscr{M}_{\lambda,}^{\mathrm{PNE}}\right)_{\sigma-\sigma_{2},-\sigma_{1} \sigma}\left(a s_{2} \mid s_{1} a\right)+\sigma_{2} u_{s_{1}} v_{s_{2}} u_{a} v_{a} \mathscr{A}_{s_{1} s_{2}}\left(\mathscr{M}_{\lambda \Omega}^{\mathrm{PNE}}\right)_{\sigma-\sigma_{1},-\sigma_{1} \sigma_{2}}\left(a a \mid s_{1} s_{2}\right) \\
& \left.\quad-\sigma_{1} u_{s_{2}} v_{s_{1}} u_{a} v_{a} \mathscr{A}_{s_{1} s_{2}}\left(\mathscr{M}_{\lambda \Omega}^{\mathrm{PNE}}\right)_{\sigma_{1}-\sigma_{2}, \sigma-\sigma}\left(s_{1} s_{2} \mid a a\right)\right\} .
\end{aligned}
$$

The antisymmetrization operator is defined as in Equation (32).

The $\mathscr{M}_{\lambda, \Omega}^{\mathrm{PNE}}$ operator connects relative $S$-states to $S$-states, which is very important because of the short range of the radial dependence ${ }^{6}$. By contrast the $V^{\mathrm{PNC}}$-admixing potentials connect relative $S$ to $P$-states. Preliminary calculations in the case of the $482 \mathrm{keV}$ line of ${ }^{181 \mathrm{Ta}}$ show that the strength of the direct transition (37) will be larger than the strength of the admixtures terms [see Eq. (11)] obtained in Ref. ${ }^{1}$ as expected.

\section{Single Particle Wave Functions}

The s.p. wave functions should be choosen as solutions of s.p. Schrödinger equation with diffuse edge deformed potential ${ }^{13}$. Except in Ref. ${ }^{1}$ until now the Nilsson-type potential has been only used, i.e. an anisotropic oscillator potential. Nevertheless, this potential together with spin-orbit and Coulomb terms which do not depend on the static nuclear deformation leads to the incorrect asymptotic behaviour of its wave functions on the nuclear surface.
Such a wrong behaviour of the wave functions leads to an inaccurate description of those nuclear phenomena for which the nuclear surface region plays an important role. This is the case for the long range electromagnetic operators we are interested in.

The diffuse edge deformed potential looks like ${ }^{13}$ :

$$
\begin{gathered}
V_{\text {s. p. }}=V_{\text {Nucl. }}+V_{\text {spin-orbit }}+V_{\text {Coul. }}, \\
V_{\text {Nucl. }}=-V_{0} n(\beta, r, \theta), \\
V_{\text {spin-orbit }}=-K \boldsymbol{p} \boldsymbol{\sigma} \operatorname{grad} V_{\text {Nucl. }}, \\
V_{\text {Coul. }}=3(Z-1) e^{2}\left(4 \pi R_{0}\right)^{-1} \\
\times \int \mathrm{d}^{3} r^{\prime} \eta\left(\beta, r^{\prime}, \theta^{\prime}\right)\left|\boldsymbol{r}-\boldsymbol{r}^{\prime}\right|^{-1}, \\
n(\beta, r, \theta)=(1+\exp [(r-R(\theta, \varphi)) / a])^{-1}, \\
R(\theta, \varphi)=R_{0}\left(1+\beta_{0}+\beta_{20} Y_{20}+\beta_{40} Y_{40}\right) .
\end{gathered}
$$

The solutions may be given numerically or approximated analytically 13 .

The new distribution of s.p. levels is different in comparison with the Nilsson one. For instance, in 
the Nilsson scheme this distribution does not depend on the mass number $A$ for the fixed deformation parameter, if the energies are given in units $\hbar \omega_{0}=41 A^{-1 / 3} \mathrm{MeV}$, the only $A$-dependence being given by this last equation. Now, such dependence, however, is not valid anymore. The dependence of the spin-orbit term on the deformation shifts the s.p. levels in a range of $\pm(0.5-0.7) \mathrm{MeV}$. While in the new model the wave functions include components from the shells situated in the vicinity of the main shell, in the Nilsson potential such components vanish. The new terms are very important in the hindered transitions as in our case. The dependence of the Coulomb term on the deformation leads to another noncoherent shift in the s.p. levels. All these facts changed very much the calculated electromagnetic transition probabilities. These quantities calculated with the potential (38) give values very near to the experimental one's while the Nilsson potential shows deviations up to a factor of $10^{3}$ (see Ref. ${ }^{18}$ ).

\section{Collective Non-rotational Structure of the Excited States}

To describe the intrinsic motion the interactions between nucleons in the nucleus are usually divided into two parts: The average nuclear field [Eq. (16)] and the residual interactions. The average field defines the bulk properties of the nucleus. The residual interactions, however, play a very important role. They are those parts of the forces which, due to their structure, cannot be included into the average field. They are not small so that one cannot calculate their effects using perturbation theory. Their effects change smoothly from nucleus to nucleus.

Because at present we are not able to take into account the residual interactions as a difference between the actual interactions among nucleons and the average field, one is forced to insert the so-called effective residual interactions [e.g. terms $H_{\text {pair }}$ $+H_{\text {long range }}$ in Eq. (18)]. However, in treating effective residual interactions we may not describe collective vibrations adiabatically. The inapplicability of the adiabatic approximation concerning individual and collective nonrotational degrees of freedom is discussed in Ref. ${ }^{11}$. This treatment is based, however, on the approximate methods, in our case the approximate second quantization ${ }^{10,11}$.
Moreover, in treating odd nuclear states we use the assumption of high collectivity [Eq. (34)], which not always may be fulfilled. In the case discussed in paper ${ }^{1}$, however, this is fulfilled. For $\beta$-vibrational phonons we inserted also the pairing vibrational terms, hence eliminating the spurious state ${ }^{11}$. The treatment mentioned above does not include the blocking effect. There is only one work ${ }^{19}$ we may use, having, however, short-comings of other character. There have been included only phonons of one type forgetting the influence of other phonons, which are very important in calculations of electromagnetic transitions. We have reglected also two phonon admixtures to the one phonon states. Also we did not change the equilibrium deformation as compared to the ground state deformation. Such methods like higher random phase approximation should be, perhaps, necessarily here to be used also ${ }^{20}$.

\section{Important Steps in Calculation of the Circular Polarization of Gamma Rays}

In the following we shall discuss some details important to be taken into account in every calculation of the circular polarization of gamma rays. This we have learned in studying ${ }^{181} \mathrm{Ta}^{1}$ and $175,177 \mathrm{Lu}^{2}$ cases.

First the strong coupling Bohr-Mottelson model can be applied because of strong deformation $\left(\beta=0.26^{10}\right)$. Then the assumption of high degree of collectivity is fulfilled. In Figs. $3-5$ we give the

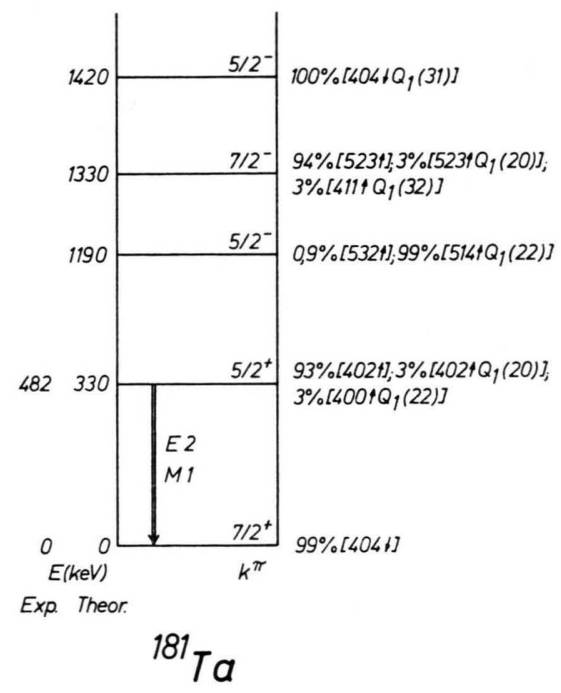

Fig. 3 a. 


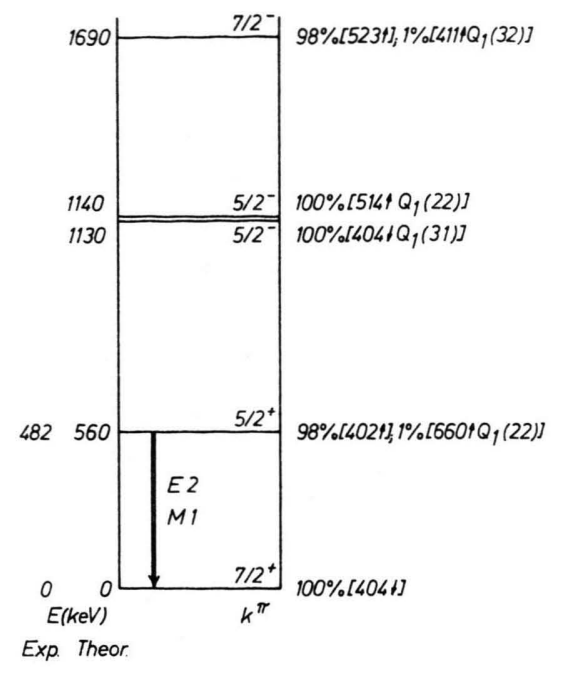

${ }^{181} \mathrm{Ta}$

Fig. 3 b.

Fig. 3. Structure and excitation energies of the level of ${ }^{181} \mathrm{Ta}$ involved in parity mixing. a) $\beta_{20}=0.26, \beta_{40}=0^{12}$; b) $\beta_{20}=0.27, \beta_{40}=-0.03^{23}$.

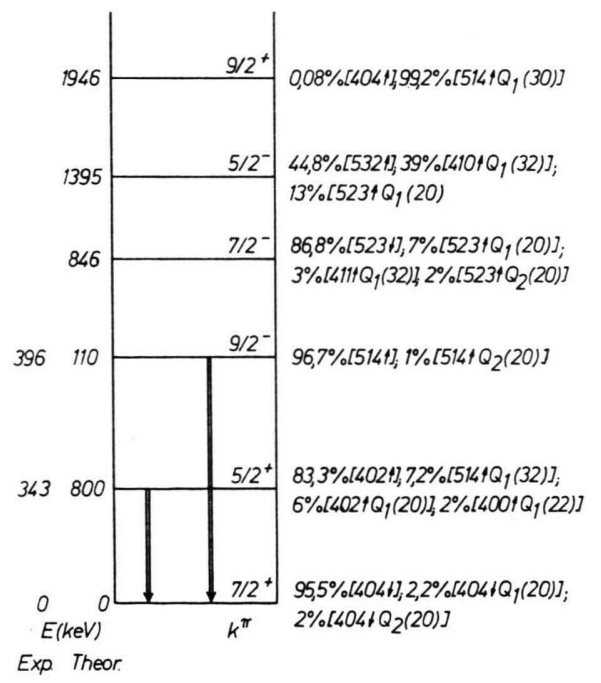

$175 \mathrm{Lu}$

Fig. 4. Structure and excitation energies of the levels of ${ }^{175} \mathrm{Lu}$ involved in parity mixing.

structure of levels taken into account in Refs. ${ }^{1,2}$ to demonstrate the situation in this region. Unfortunately the higher opposite parity excited states have not yet been found experimentally. We believe, however, more in the pairing plus quadrupole and octupole model than in the simple pairing model ${ }^{21}$. The first one has explained a lot of experimental

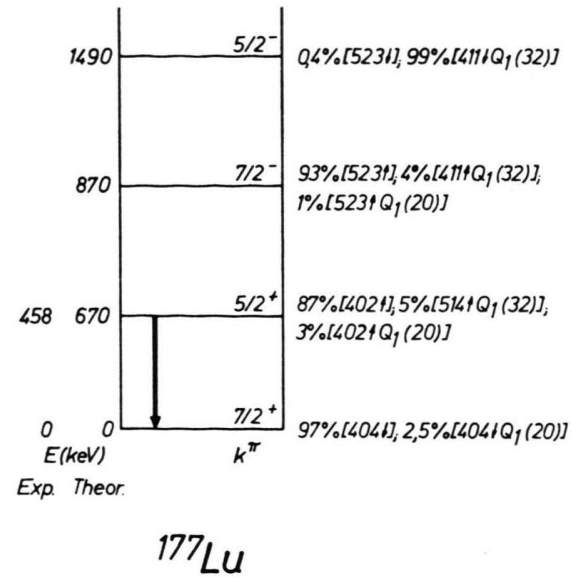

Fig. 5. Structure and excitation energies of the levels of ${ }^{177} \mathrm{Lu}$ involved in parity mixing.

data in the Ta-region; therefore the complex structure of the states cannot be ignored. While in ${ }^{21}$ the excited states are pure one or three quasiparticle states, our picture gives a much more differentiated structure. Because of the high collectivity (see Table 1 and 2 for $\mathrm{Q}(22)$ and $\mathrm{Q}(31)$ phonons; for other phonon contributions (Figs. $3-5$ ) the collec-

Table 1. Two quasiparticle states occuring in the $\mathrm{Q}(22)$. phonon of the $1190 \mathrm{keV}-\frac{5-}{2}$ state of $181 \mathrm{Ta}$, which give nonzero contributions to the PNC-matrix elements [see Eq. (23) and Figure 3].

\begin{tabular}{llll}
\hline \multicolumn{2}{l}{ neutron states } & $10^{3} \psi_{s s t}^{i}$ & $10^{3} \varphi_{s s "}^{i}$ \\
$s$ & $s^{\prime}$ & & \\
\hline $523 \downarrow$ & $521 \downarrow$ & -51 & -31 \\
$521 \downarrow$ & $512 \uparrow$ & +13 & +6 \\
$512 \uparrow$ & $510 \uparrow$ & -615 & -208 \\
$514 \downarrow$ & $512 \downarrow$ & -791 & -197 \\
$514 \downarrow$ & $501 \uparrow$ & +8 & +3 \\
$512 \downarrow$ & $503 \uparrow$ & +25 & +8 \\
$503 \uparrow$ & $501 \uparrow$ & -274 & -120 \\
$505 \uparrow$ & $503 \uparrow$ & -137 & -93 \\
$642 \uparrow$ & $651 \downarrow$ & +46 & +28 \\
$642 \uparrow$ & $640 \uparrow$ & -96 & -60 \\
$651 \uparrow$ & $633 \uparrow$ & -18 & -12 \\
$642 \uparrow$ & $624 \uparrow$ & -66 & -36 \\
$633 \uparrow$ & $615 \uparrow$ & -60 & -33 \\
$521 \uparrow$ & $521 \downarrow$ & -50 & -32 \\
$512 \downarrow$ & $510 \uparrow$ & -765 & -193 \\
$651 \uparrow$ & $651 \downarrow$ & -61 & -40 \\
\hline proton states & & \\
$402 \uparrow$ & $411 \downarrow$ & -64 & -15 \\
$402 \uparrow$ & $400 \uparrow$ & +296 & +123 \\
$404 \uparrow$ & $402 \uparrow$ & +152 & +95 \\
\hline
\end{tabular}


Table 2. Two quasiparticle states occuring in the $Q(31)$ phonon of the $1420 \mathrm{keV}-{ }_{2}^{-}$state of $181 \mathrm{Ta}$, which give nonzero contributions to the PNC-matrix elements [see Eq. (23) and Figure 3].

\begin{tabular}{llll}
\hline \multicolumn{2}{l}{ neutron states } & $10^{3} \psi_{s s t}^{i}$ & $10^{3} \varphi_{s s \prime}^{i}$ \\
$s$ & $s^{\prime}$ & & \\
\hline $651 \downarrow$ & $512 \downarrow$ & -5 & -2 \\
$640 \uparrow$ & $521 \uparrow$ & -12 & -7 \\
$624 \uparrow$ & $514 \downarrow$ & -26 & -5 \\
$624 \uparrow$ & $503 \uparrow$ & +37 & +8 \\
$633 \uparrow$ & $512 \uparrow$ & +7 & +3 \\
$651 \downarrow$ & $770 \uparrow$ & -6 & -3 \\
$640 \uparrow$ & $521 \downarrow$ & +24 & +12 \\
$651 \downarrow$ & $510 \uparrow$ & +6 & +2 \\
$640 \uparrow$ & $770 \uparrow$ & +2 & +1 \\
$660 \uparrow$ & $761 \uparrow$ & -11 & -8 \\
$642 \downarrow$ & $521 \downarrow$ & +13 & +8 \\
$642 \downarrow$ & $523 \downarrow$ & -10 & -7 \\
\hline
\end{tabular}

proton states

$402 \uparrow \quad 523 \uparrow \quad-20 \quad-7$

tivity is much higher ${ }^{22}$ ) of many levels the PNC matrix elements decrease compared to 21 . This effect is of second order if we look at the reduced matrix elements of regular electromagnetic transitions [see the last two terms in Eq. (11)].

Of course higher excited may contribute also; however we believe in the high collectivity of these levels; therefore no important contribution could arise. No three-quasiparticle states having $K \pi=5^{-/ 2}$ or $7^{-/ 2}$ in ${ }^{181} \mathrm{Ta}$ and ${ }^{175} \mathrm{Lu}$ occur in this pairing + quadrupole + octupole model. We may learn from Ref. 23 that the three-quasiparticle states are characterized by high spins (more than 15/2). Therefore we may not expect this structure to occur in our cases. Here we want to mention that the phonon structure (e.g. Tables 1,2) is calculated ${ }^{22}$ using a Nilsson model; however, this structure is not significantly changed by passing to the diffuse edge potential. It is also important to know which strength parameters $G_{\varrho}$ and $X_{e e}^{(1)}$ have to be taken into account. Especially $X_{\rho \rho^{\prime}}^{(\lambda)}$ are taken to be

$$
\begin{aligned}
& X_{n n}^{(2)}=X_{n p}^{(2)}=X_{p p}^{(2)}=2.0 \quad A^{-4 / 3} \mathrm{MeV} / \mathrm{cm}^{4}, \\
& X_{n n}^{(3)}=X_{n p}^{(3)}=X_{p p}^{(3)}=0.04 A^{-4 / 3} \mathrm{MeV} / \mathrm{cm}^{6}
\end{aligned}
$$

for a large number of nuclei 22 . However, this may be not as good when the Nilsson potential is replaced by the diffuse egde one. Unfortunately this aspect is not discussed even in the recent paper ${ }^{23}$. In s pite of the fact that the accuracy in calculating the wave functions (25) is restricted, however, the method we use is much more realistic than to neglect the long range terms (26).

In applying the diffuse edge model (38) we got great differences for allowed electromagnetic transitions as compared with Nilsson ones. The same results are given in Ref. ${ }^{18}$. Therefore we concluded that the Nilsson model should not be used in calculating the $V_{\tau \tau^{\prime} \sigma \sigma^{\prime}}^{\mathrm{PNC}}\left(v v^{\prime} \mid \omega \omega^{\prime}\right)$ and $\left(\mathscr{M}_{\lambda \Omega}^{\mathrm{PNE}}\right)_{\tau \tau^{\prime} \sigma \sigma^{\prime}}\left(v v^{\prime} \mid \omega \omega^{\prime}\right)$ together with the regular electromagnetic matrix elements.

The accurate treatment of the short range correlations according to Refs. ${ }^{3,12}$ shows that the polarization is strongly suppressed by the strong interaction. Regarding PNC contributions the $\varrho$-exchange contribution are about 6 times smaller than without correlations ${ }^{1}$. Also the pion-exchange contribution is diminished 1,2 by a factor of $2-3$. This is in complete agreement with our earlier calculations 12, 14,16. Similar trends are found in Ref. ${ }^{21}$; however, the short range correlations have been either roughly estimated only or a simple model calculation has been performed ${ }^{24}$. It is important to note that one is not allowed to take SRC's from the n-p system. There is no ,healing“" in a two body system, whereas this effect becomes extremely important for heavy nuclei. As is well known, ,healing" is due to the presence of many other nucleors in occupied levels and therefore increases with $\mathrm{A}$. One even should not take over SRC's from light nuclei to heavy ones.

In Fig. 6 we give examples for the realistic wave function in ${ }^{181} \mathrm{Ta}^{1}$ for the Reid soft core potential in comparison to the ${ }^{16} \mathrm{O}$ case ${ }^{14}$.

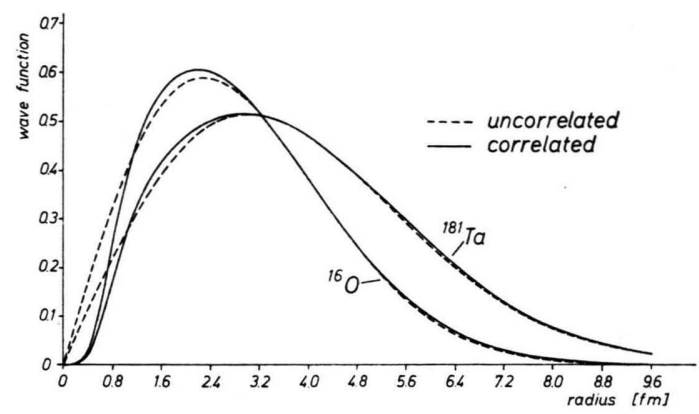

Fig. 6. Relative $S$ wave function for ${ }^{181} \mathrm{Ta}$ and ${ }^{16} \mathrm{O}$ using the Reid soft core potential.

Taking into account Eqs. (11), (27), (30), (31) and (37) together with (2) we may claim that such calculations like in Refs. ${ }^{25,26}$ are meaningless. We are not allowed to factorize the polarization in the form

$$
P=-2 /\left(1+\delta^{2}\right) F R
$$

as is done in 25,26 . 


\section{Conclusions}

Explicit expressions for the PNC and PNE matrix elements occuring in the formula for the circular polarization of gamma rays, as emitted by odd mass deformed nuclei, are given. The theory is developed in the framework of the strong coupling BohrMottelson-model. Semi-microscopic pairing plus quadrupole and octupole description is used to describe the parity conserving $|K \pi\rangle$-internal excited states. Single particle wave functions of the diffuse edge deformed potential have to be used.

Short range correlations are taken into account using the generalized Bethe-Goldstone method, and not some rough descriptions like the Jastrow model or extrapolations of results obtained for simple systems like the free np system.

There is no difficulty to get expressions similar to Eqs. (30), (31) and (37) in the case of a double even deformed nucleus. However, there are only experiments on ${ }^{180} \mathrm{Hf} 27,28$, where the strong coupling Bohr-Mottelson model (5) is not applicable anymore. A strong Coriolis interaction has to be added.

Concerning the numerical calculations on ${ }^{181} \mathrm{Ta}$ and ${ }^{176} \mathrm{Lu} 1,2,24$ it is important to note that they

1 M. Gari, O. Dumitrescu, J. G. Zabolitzky, and H. Kümmel, Phys. Letters 35 B, 19 [1971].

2 O. Dumitrescu, M. Gari, H. Kümmel, and J. G. ZaboLITZKx, Preprint Bochum.

3 H. Kümmel, Theory of Many Body Wave Functions with Correlations, Nucl. Phys. A 176, 205 [1971].

4 H. KüMmeL, Calculation of Parity Violating Effects in Nuclei, Lecture given at the Symposium on Nucleons and Weak Interactions, Zagreb/Yugoslavia, 7-11 July 1971. Preprint Ruhr-Universität Bochum, TP II/29 [1971], to be published.

5 L. ScHülke, Nucl. Phys. B 40, 386 [1972].

6 M. Gari and A. H. Huffman, Phys. Letters 36 B, No 5 442 [1971].

7 See Proceedings of the Symposium on Nucleons and Weak Interactions, July 1971, Zagreb, Yugoslavia.

8 M. Gari, A. Huffman, to be published.

9 A. Bohr, and B. Mottelson, Mat. Fys. Medd. Dan. Vid. Selsk 27, Nr. 16 [1953].

10 L. A. Malov, V. G. Soloviev, and U. M. Fainer, Izv. A.N. SSSR (serv. fiz.) 33, 1244 [1969].

11 V. G. Soloviev, Atomic Energy Rev. 3, 117 [1965]; Nucl. Phys. 69, 1 [1965].

12 M. Gari, H. Kümmel, and J. G. Zabolitzky, Nucl. Phys. A 161, 625 [1971].

13 F. A. Gareev and S. P. Ivanova, Preprint JINR-Dubna P4-5221 [1970]; - F. A. Gareev, S. P. Ivanova, B. N. Kalinkin, S. K. Slepnev, and M. G. Ginsburg, Preprint JINR-Dubna P4-3607 [1967].

14 M. GARI, Phys. Letters 31 B, 627 [1970]; Z. Physik 231, 412 [1970].

15 O. Dumitrescu and A. Sandulescu, Nucl. Phys. A100, 456 [1967]. did not include the new direct (Fig. 2) parity violating photon emission terms (as everybody else did), which are required by gauge invariance ${ }^{6}$. Thus, the results obtained there were too small, since they included only contributions due to parity admixtures. It is hoped that calculations including these new terms will yield agreement with experiments. The calculations are not easy to perform and require a computer time comparable with the immense time needed. e.g. in Refs. ${ }^{1,2}, 1578$ hrs. of computer time on the Telefunken TR 440 have been used.

Also we should note that first order perturbation theory with respect to electromagnetic forces may not be sufficient for nuclei with large proton numbers like Ta. One could try to do higher order calculations by introducing resonant states of the A nucleon system plus the electromagnetic field in the spirit of the formal decay theory, see Refs. ${ }^{29,30 .}$

The calculations have been performed on the TR 440 of the "Rechenzentrum der Ruhr-Universität Bochum". We feel obliged to thank especially H. P. Zoller for good cooperation. We also thank for the kind hospitality extended to two of us (M.G. and O.D.) by Professor A. BoHr and for most helfpul discussions with Professor G. E. Brown. One of us (O.D.) is grateful to the Alexander von HumboldtFoundation for a fellowship.

16 U. M. Wambach ,M. Gari, and H. Kümmel, Phys. Letters 33 B, 253 [1970].

17 In all cases under consideration this seems to be a good approximation since only low lying levels are involved (see Refs. ${ }^{1,10}$ ).

18 H. Schulz and H. J. Wiebicke, Preprint JINR-Dubna E4-4210 [1968].

19 D R. Bes and Chо YI-Ching, Nucl. Phys. 86, 581 [1966]

20 A. Raduta, A. Sandulejcu, and P. O. Lipas, Nucl. Phys. A 149, 11 [1970].

21 B. Desplangues and N. Vinh Mau, Phys. Letters 35 B, 28 [1971].

22 K. M. Zheleznova, A. A. Korneichuk, V. G. Soloviev, P. Vogel, and G. Yungklaussen, Preprint JINRDubna D-2157 [1965].

23 V. G. Soloviev and U. M. Fainer, Preprint JINRDubna, E4-6030 [1971], to be published in Izv. A.N. SSSR (Ser. Fiz.).

24 N. Vinh MaU, Lecture given at the Symnosipm on Nucleons and Weak Interactions, Zagreb, Yugoslavia 1971.

25 J. Grabowski, Phys. Letters 33 B, 268 [1970].

26 M. E. Voychanski and M. A. Listengarten, Izv. ANSSSR (Ser. Fiz.) 33, 98 [1969].

27 B. Jenschke and P. Bock, Phys. Letters 31 B, 65 [1970].

28 E. D. Lipson, F. Boeny, and J. C. VANderleeden, Phys. Letters 35 B, 307 [1971].

29 M. L. Goldberger and K. M. Watson, "Collective Theory", John Wiley \& Sons, Inc., New York-LondonSydney [1964], Chap. (8.3).

30 O. Dumitrescu and H. Künmel, "Resonance Scattering Theory and the Structure of Compound States". Preprint, Ruhr-Universität Bochum, TP II/27 [1971]. to be published in Ann. Physics, New York. 